\title{
Uma Infraestrutura de Rede Desconectada Colaborativa Oportunística para Mediar a Interação Professor/Aluno
}

\author{
Jackanderson C. L. de Menezes ${ }^{1}$, Jonas D. R. Martins ${ }^{2}$, Cidcley T. de Souza ${ }^{1}$ \\ ${ }^{1}$ Programa de Pós-Graduação em Ciência da Computação (PPGCC) \\ Instituto Federal do Ceará (IFCE) \\ Av. 13 de Maio 2081, Benfica - 60040-215 - Fortaleza - CE - Brasil \\ ${ }^{2}$ Secretaria Municipal de Educação \\ Prefeitura de Santa Quitéria \\ Av. José Emídio 134, Boa Vida - 62.280-000 - Santa Quitéria - CE - Brasil

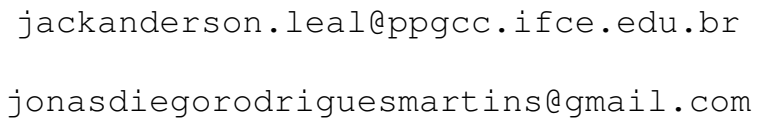

\begin{abstract}
This paper presents a new approach to enable the interaction between teachers and students through a disconnected network infrastructure supporting opportunistic collaboration, called PWNet. A PWNet is an adhoc network infrastructure that can hierarchically connect devices such as smartphones, tablets and notebooks from a PWA (Progressive Web App) allowing them to form an access network without the need for an Internet connection. To show the benefits of a PWNet in education, a case study is presented in which an "MyQuiz"app was developed that is deployed as a PWA on a teacher's smartphone, and even without an Internet connection, students can interact and multiply access to other students.
\end{abstract}

Resumo. Este trabalho apresenta uma nova abordagem para viabilizar a interação entre professores e alunos através de uma infraestrutura de rede desconectada da Internet de suporte à colaboração oportunística, denominada PWNet. Uma PWNet é uma infraestrutura de rede adhoc que pode conectar hierarquicamente equipamentos tais como smartphones, tablets e notebooks, a partir de uma PWA (Progressive Web App) permitindo que os mesmos possam formar uma malha de acesso sem a necessidade de conexão com a Internet. Para mostrar os benefícios de uma PWNet em âmbito educacional, o artigo apresenta um App denominado "MyQuiz"que é implantado como uma PWA no smartphone de um professor e, mesmo sem conexão com a Internet, os alunos podem interagir e multiplicar os acessos para outros alunos.

\section{Introdução}

A utilização de dispositivos móveis no contexto das salas de aula vem sendo investigada em várias pesquisas nos últimos anos [Bastos and Rapkiewicz 2017]. Diversos benefícios podem ser apontados na literatura, sendo que tanto aplicativos específicos de interação, ou outros genéricos, como o Whatsapp [Junior et al. 2015], estão sendo adotados com sucesso no suporte ao ensino-aprendizagem. 
VII Congresso Brasileiro de Informática na Educação (CBIE 2018)

Anais do XXIX Simpósio Brasileiro de Informática na Educação (SBIE 2018)

Esse cenário ainda é mais promissor se for observado a vasta difusão de smartphones entre a população brasileira. Em pesquisa recente [PNAD 2016], o IBGE indica que que $77,1 \%$ da população, com 10 anos ou mais de idade, tem um aparelho de celular próprio, sendo a grande maioria smartphone. Observando o aspecto de acesso a Internet, a mesma pesquisa indica que $94,6 \%$ das pessoas se conectaram via celular.

No contexto educacional, essas estatísticas contrastam com os dados do Censo Escolar 2017 [MEC 2017], divulgado pelo Ministério da Educação (MEC), que constata que a tecnologia não está acessível aos estudantes em cerca da metade das escolas de ensino fundamental.

Além disso, ainda segundo o IBGE, o principal motivo para a não utilização da Internet é o seu alto custo, que chega $29 \%$, sendo que essa estatística chega a 34,8\% na região nordeste.

Observando esse cenário, o objetivo dessa pesquisa é propor uma infraestrutura de rede que possa viabilizar a colaboração em sala de aula sem a necessidade de acesso à Internet. Essa rede, denominada PWNet, utiliza as recentes Progressive Web Apps como mecanismo de apoio à disseminação de acesso colaborativo entre smartphones dentro de uma mesma rede WiFi, sem a necessidade de instalação de nenhum software e nem de acesso à Internet. Com isso, alunos e professores poderão realizar diversas atividades colaborativas usando seus smartphones com o mínimo de requisitos de infraestrutura.

Para apresentar essa proposta, esse artigo está organizado, além dessa seção, da seguinte forma. Na seção 2 são apresentados os principais trabalhos relacionados. Na seção 3 a PWnet é apresentada. Na seção 4 é apresentado um exemplo que demonstra os benefícios da PWnet. Para finalizar, a seção 5 apresenta algumas conclusões e trabalhos em andamento.

\section{Trabalhos Relacionados}

Existem vários aplicativos que foram desenvolvidos para permitir a colaboração em sala de aula. [Almeida et al. 2018] desenvolveu um jogo similar a um Quiz, com perguntas e respostas denominado de "SuperQuiz". Esse App, desenvolvido em android, precisa estar instalado em todos os dispositivos interessados em interagir.

[Abreu et al. 2017], apresenta o aplicativo Fantastic Pirates (FP), um Quiz multidisciplinar, desenvolvido para crianças de até 10 anos, onde os educadores elaboram perguntas e criam feedbacks. O aplicativo foi desenvolvido para ser usado em smartphones de sistema operacional android, e deve acessar informações em um servidor na Internet.

O FireChat [Simonite 2014] é um aplicativo desenvolvido para sistemas Android e iOS que permite pessoas que estejam próximas se comunicarem através de uma rede. Para que haja interação entre os dispositivos todos os smartphones precisam baixar e instalar o App da Internet.

\section{PWNet}

Uma PWNet deve funcionar como uma rede em malha sem fio, conectando dispositivos móveis próximos e permitindo a interatividade entre eles. Organizada de modo hierárquico, conforme mostra a Figura 1, essa rede é viabilizada através de funções implementadas dentro de uma PWA. 
VII Congresso Brasileiro de Informática na Educação (CBIE 2018)

Anais do XXIX Simpósio Brasileiro de Informática na Educação (SBIE 2018)

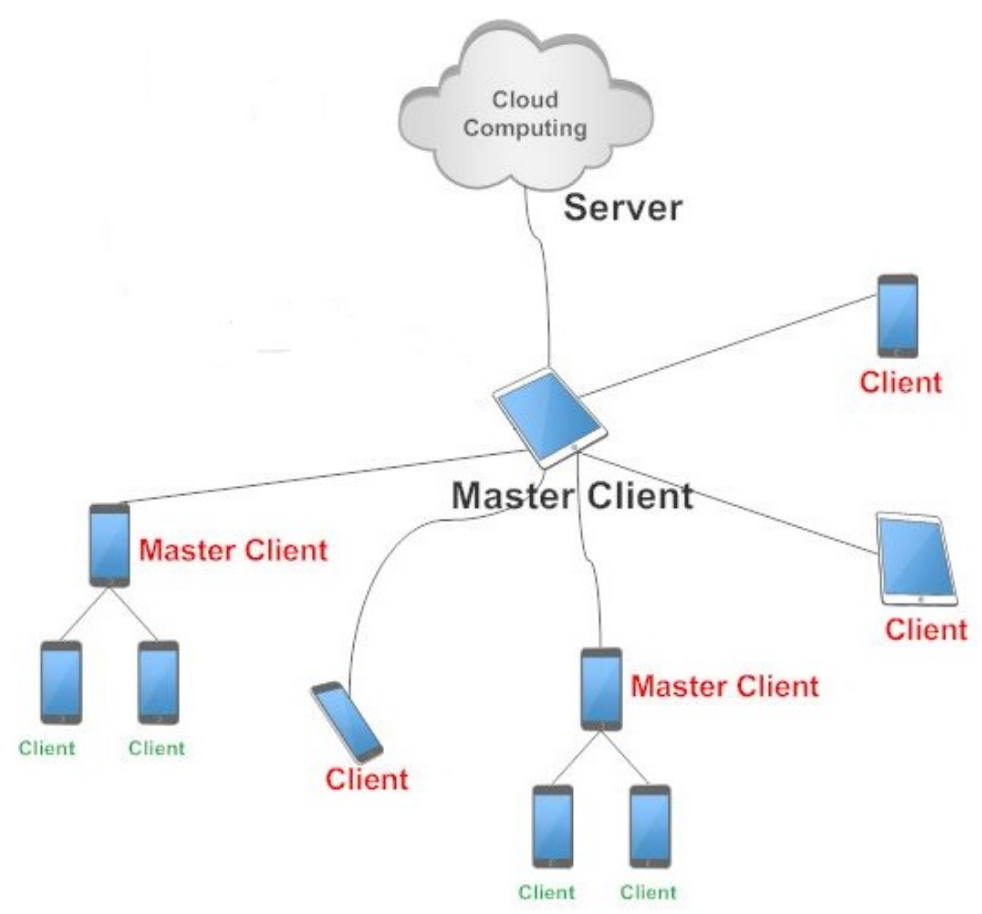

Figura 1. Infraestrutura de uma PWNet

PWAs são aplicativos web que possuem comportamento e aparência semelhantes a um aplicativo nativo. Como se fosse um aplicativo instalado em um smartphone, acessa recursos do dispositivo da mesma forma que as aplicações nativos, também usa o Service Workers que possibilitam o acesso à aplicação off-line, receber push de notificações, atualizar conteúdos em segundo plano, dentre outras funcionalidades [Hume 2017]

Dessa forma, para que uma PWNet seja construída, a aplicação do usuário, como por exemplo uma aplicação de Quiz, um Chat, ou um sistema colaborativo mais complexo, deve ser implementada como uma PWA com algumas características específicas que dotarão essa PWA com as funcionalidades requeridas para a viabilização da PWNet.

Essa PWA deve ter instalada um Service Worker que incluirá os códigos de um servidor web mínimo que ficará acessível quando essa aplicação tiver offline. Desse modo, os códigos em questão possibilitarão o acesso de outras aplicações à PWA que, ao estar offline, se comportará como um servidor web e tornará os próprios arquivos da PWA acessíveis e, dessa forma, propagará a PWA para outros dispositivos.

Dessa forma, a PWNet deve ser iniciada por um dispositivo (notebook, PC, smartphone) que em algum momento acessou a Internet e realizou a implantação, via browser, da PWA. Ou seja, não é necessário realizar explicitamente nenhuma instalação. Dessa forma, o Cliente passa a ter a PWA.

Estando dentro de uma mesma rede WiFi, esse equipamento pode passar a fornecer acesso à PWA, que foi implementada para funcionar internamente como um Servidor Web, para viabilizar a implantação da mesma aplicação em outros dispositivos, como mostra a Figura 1. Neste momento, a PWA se tornará um Cliente Master, indicando que o mesmo estará servindo a aplicação para outros clientes dentro da mesma rede. 
VII Congresso Brasileiro de Informática na Educação (CBIE 2018)

Anais do XXIX Simpósio Brasileiro de Informática na Educação (SBIE 2018)
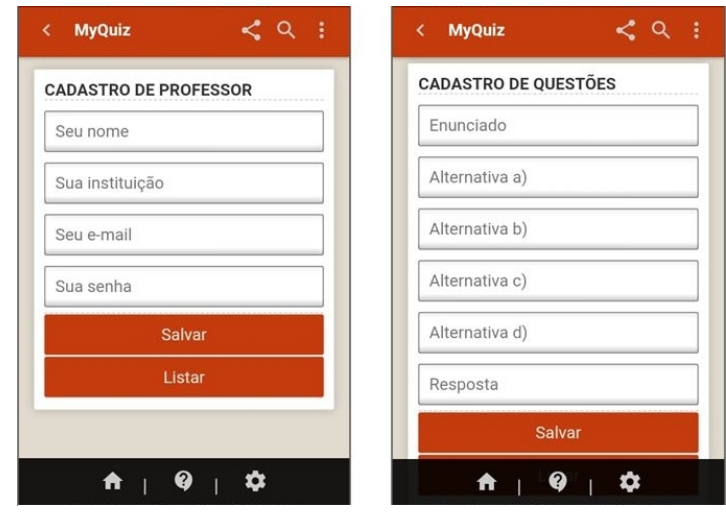

(a) Tela cadastro do Professor. (b) Cadastro de questões.

Figura 2. Telas do "MyQuiz"para o professor

\section{A Aplicação "MyQuiz"}

Para exemplificar a construção de uma PWNet e demonstrar sua utilização no contexto educacional, é apresentado aqui o "MyQuiz". Esta aplicação disponibiliza, via PWA, uma ferramenta de Quiz na qual professor e alunos podem interagir. Inicialmente o professor acessa via Web a PWA do MyQuiz, fazendo a implantação da mesma via browser.

Por ser construída para dar suporte à PWNet, essa aplicação fica disponível para acesso pelos os alunos a partir da máquina do professor sem a necessidade de realizar nenhuma instalação de software adicional para esse fim. Assim, o professor faz o login na aplicação (Figura 2(a)) para ter acesso ao gerenciamento do Quiz, ao cadastro dos alunos e ao cadastro das perguntas (Figura 2(b)) a serem respondidas pelos alunos.

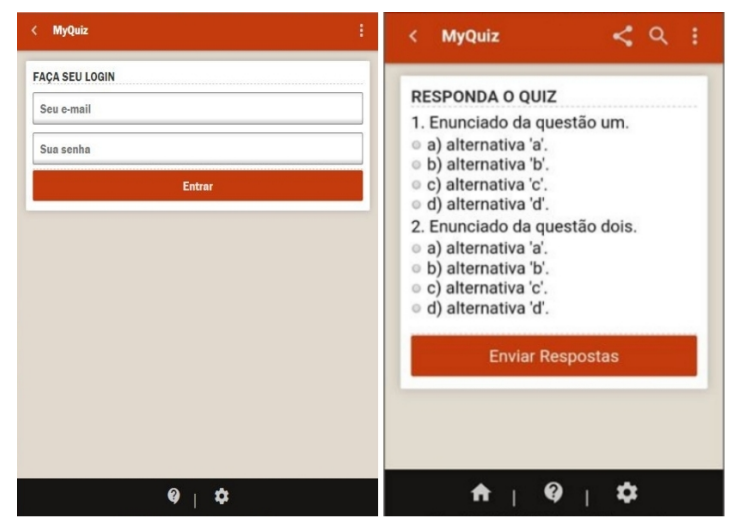

(a) Login dos alunos. (b) Responder questões.

Figura 3. Telas do "MyQuiz"para os alunos

Posteriormente, os alunos poderão acessar a máquina do professor, que deverá ligar o roteador $W i F i$ e, dessa forma, ficar acessível para os dispositivos na mesma rede. Desse modo, os alunos poderão acessar, via browser, o MyQuiz que estará disponibilizado no endereço do dispositivo do professor. Sendo possível realizar o login (Figura 3(a)) e realizar as respostas das perguntas (Figura 3(b)).

Sendo uma PWA, os alunos também poderão implantar o MyQuiz nos seus dispositivos e, caso queiram, desconectar da rede e responder as questões posteriormente. Em 
VII Congresso Brasileiro de Informática na Educação (CBIE 2018)

Anais do XXIX Simpósio Brasileiro de Informática na Educação (SBIE 2018)

um momento futuro, quando os alunos voltarem a se conectar à rede, as respostas serão sincronizadas pela PWA com a aplicação do professor quando ambos estiverem na mesma rede novamente.

\section{Conclusões}

A proposta da PWNet, apresentada nesse artigo, considera que a interação entre professores e alunos pode ser viabilizada com o mínimo de infraestrutura possível. Assim, com smartphones conectados a uma rede $\mathrm{WiFi}$, esses dispositivos podem interagir usando aplicativos que podem ser trocados entre si, sem a necessidade de instalação prévia a partir da Internet.

Atualmente está sendo implementada uma versão estável dos códigos a serem disponibilizados pelos Service Workers de PWAs de modo a fornecer o suporte necessário para que qualquer PWA dê suporte a uma PWNet. Também será realizada uma avaliação sobre as limitações em termos de funcionalidades, decorrentes das limitações das PWAs, para as aplicações passíveis de funcionar em uma PWNet.

\section{Referências}

Abreu, C., Rosa, J., and Matos, E. (2017). Fantastic pirates: software de apoio ao ensino e à aprendizagem infantil. In Anais dos Workshops do Congresso Brasileiro de Informática na Educação, volume 6, page 252.

Almeida, T., de Andrade, M., Santos, J., and Gadelha, B. (2018). Superquiz: Um jogo colaborativo baseado em quiz para dispositivos móveis. Anais do Computer on the Beach, pages 100-109.

Bastos, J. A. R. and Rapkiewicz, C. E. (2017). Dispositivos móveis e redes sociais no contexto de letramento digital comunicacional.

Hume, D. A. (2017). Progressive Web Apps. Manning Publications Co., Greenwich, CT, USA, 1st edition.

Junior, J. C. d. S. F., Saccol, A. Z., da Silva, J. V. V. M., Barbosa, J. L. V., and Baldasso, L. (2015). O uso do aplicativo whatsapp $\AA$ como recurso de m-learning no ensino e aprendizagem em cursos de administração.

MEC (2017). Censo escolar 2017. Disponível em: http://www.censobasico.inep.gov.br. Acesso em agosto de 2018.

PNAD, I. (2016). Pesquisa nacional por amostra de domicílio - pnad - acesso à internet e à televisão e posse de telefone móvel celular para uso pessoal: 2015. Coordenação de Trabalho e Rendimento. Rio de Janeiro. Disponível em: $<$ http://biblioteca.ibge.gov.br/visualizacao/livros/liv95753.pdf $>$. Acesso em agosto de 2018.

Simonite, T. (2014). Firechat could be the first in a wave of mesh networking apps. MIT Technology Review. 